\title{
CASE REPORT \\ Emergency surgery in a patient with large spontaneous spinal epidural hematoma determining excellent neurological recovery: review of the literature
}

\author{
A Giugno, L Basile, R Maugeri and DG Iacopino
}

\begin{abstract}
Study design: Case report.
Objectives: We report a case of a 75-year-old woman suffering from voluminous idiopathic spontaneous spinal epidural hematoma (SSEH) that was rapidly diagnosed and successfully treated.

Methods: Clinical presentation was characterized by sudden and intense back pain that rapidly evolved into plegia of the right leg and severe paresis of the left leg. Hypoesthesia below T6 and urinary retention were also present. Magnetic resonance imaging showed a significant posterior spinal compression from T6 through L3 caused by an epidural hematoma that involved 10 metameric levels, extending for $\sim 20 \mathrm{~cm}$, with a maximum thickness of $1.6 \mathrm{~cm}$ from T12 to L1.

Results: Within $12 \mathrm{~h}$, emergency decompressive laminectomy from T10 to L1 was performed, and evacuation of the hematoma was achieved. The postoperative course and neurological recovery of the patient were optimal. After discharge, the patient continued the rehabilitative treatment started during hospitalization, achieving an excellent functional outcome in 1 month.

Conclusions: Spinal epidural hematoma (SEH) is a rare clinical finding that can occur following trauma or spontaneously (SSEH). We describe, to the best of our knowledge, the second most extensive idiopathic SSEH and the longest with involvement of the dorsolumbar spine that had a excellent functional outcome due to emergency decompressive laminectomy, which is emphasized in the treatment of these rare pathologies.
\end{abstract}

Spinal Cord (2014) 52, S22-S24; doi:10.1038/sc.2014.156

\section{INTRODUCTION}

Even though spinal hematoma was first reported in the mid-19th century, ${ }^{1}$ it remains a rare clinical finding that can occur following trauma or spontaneously. Epidural spinal hematomas represent $75 \%$ of all spinal hematomas and can be associated with tumors, vascular malformations or pharmacological causes, with $\sim 30 \%$ of cases arising idiopathically. ${ }^{2}$ It is estimated that spontaneous spinal epidural hematomas (SSEHs) arise in $\sim 0.1$ patient in every 100000 patients, ${ }^{3}$ with a male-female ratio of 1.4:1. ${ }^{4}$ The clinical presentation varies depending on the spinal level affected, and can evolve very quickly from minor symptoms to a complete neurological deficit. In most cases, only early diagnosis with urgent treatment can allow the recovery of neurological function, sometimes even being vital for survival. ${ }^{5,6}$ We report a case of a voluminous idiopathic SSEH that was rapidly diagnosed and successfully treated.

\section{CASE REPORT}

A 75-year-old woman presented with a sudden and intense back pain that started in the morning, and rapidly evolved to weakness of the lower limbs, especially the right one, accompanied by difficulty in urination. By mid-afternoon, when she was brought to our department, her condition had progressed to a severe paresis, rapidly evolving into plegia of the right leg and severe paresis of the left leg.
Hypoesthesia below T6 and urinary retention were also present (Frankel grade B). The patient reported no pre-existing pathological conditions, no past medications, nor history of trauma. Blood chemistry analyses excluded coagulation problems and all other parameters were also normal. Magnetic resonance imaging showed a significant posterior spinal compression from T6 through L3. The lesion appeared inhomogeneous on T2-weighted sequences, and slightly isointense on T1-weighted sequences, and extended for $\sim 20 \mathrm{~cm}$ along the epidural space, with a maximum thickness of $1.6 \mathrm{~cm}$ from T12 to L1 (Figures 1a-c). Contrast medium application excluded a neoplastic origin of the lesion, confirming the suspicion of an idiopathic epidural hematoma. In the same evening, with an extremely short operative interval $(<12 \mathrm{~h})$, emergency decompressive laminectomy from T10 to L1 was performed with evacuation of the hematoma. The collection of blood was for the most part coagulated, and no particular difficulty was encountered in obtaining hemostasis. The dura mater appeared normal, and no active hemorrhage was observed within the subdural space. No vascular malformations or tumors were detected during surgery. The following day, the patient underwent a follow-up magnetic resonance imaging that confirmed the nearly complete removal of the hematoma and an excellent level of spinal decompression, without any evidence of surgical complications (Figures $2 \mathrm{a}$ and $\mathrm{b}$ ). The postoperative course and neurological 
a

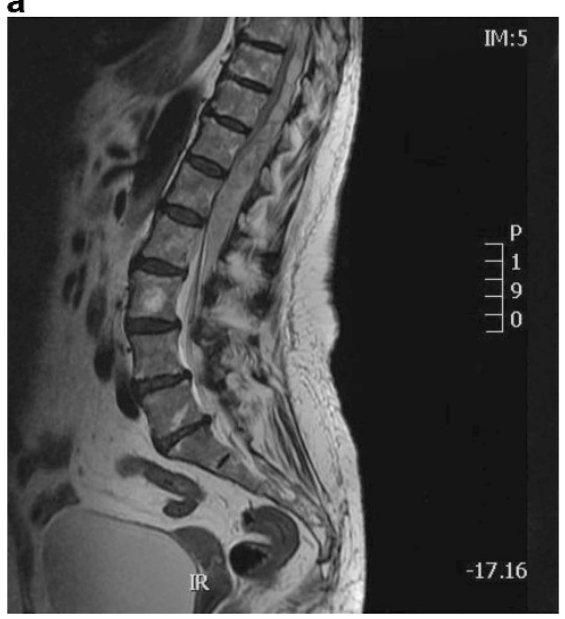

b

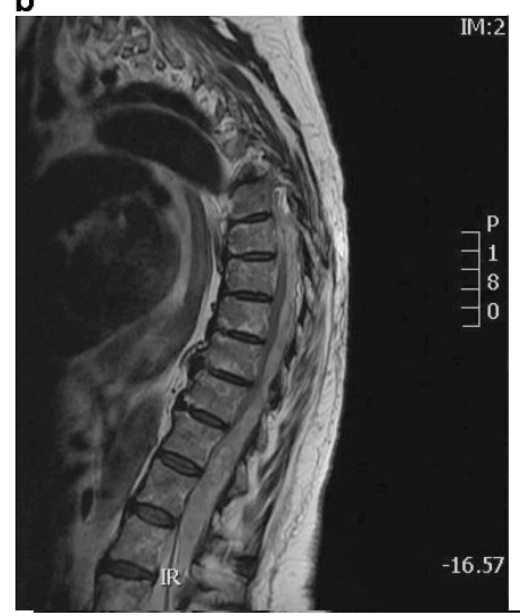

c

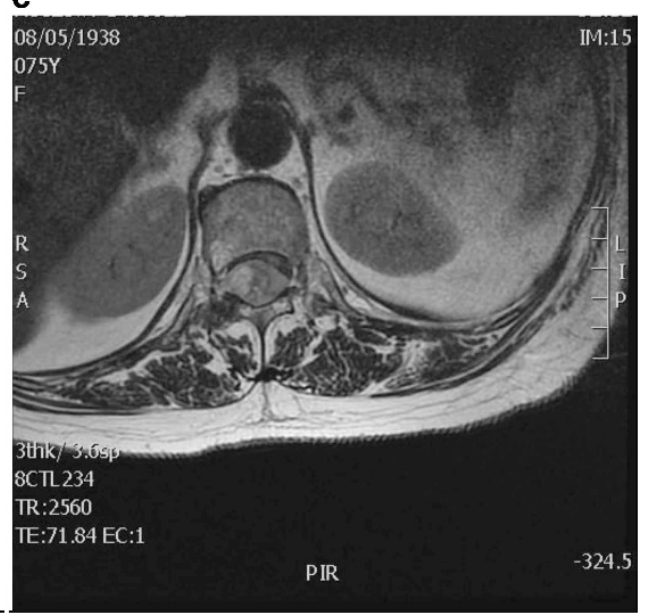

Figure 1 Preoperative magnetic resonance and slightly inhomogeneous epidural spinal hematoma on T2-weighted sequences, extending from T6 to L3 for $\sim 20 \mathrm{~cm}$ along the epidural space $(\mathbf{a}, \mathbf{b})$, with a maximum thickness of $1.6 \mathrm{~cm}$ from 12 to $\mathrm{L} 1(\mathbf{a}, \mathbf{c})$.

a

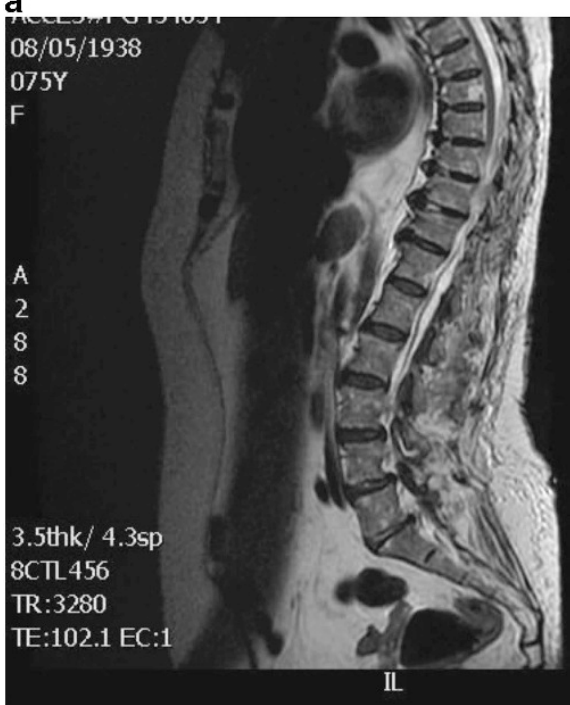

b

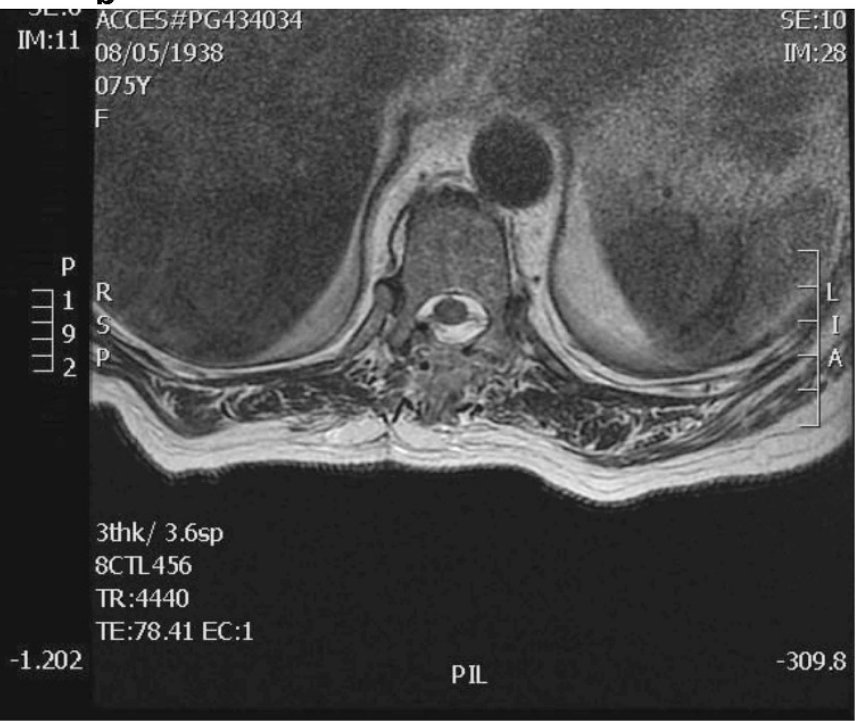

Figure 2 Postoperative magnetic resonance, nearly complete removal of the hematoma (a) and an excellent level of spinal decompression without any evidence of surgical complications (b).

recovery of the patient were optimal, with only mild residual paresis of the right leg, while the left leg recovered fully (Frankel grade D). No medical complications occurred following the surgery. After discharge, the patient continued the rehabilitative treatment started during hospitalization, achieving an excellent functional outcome in 1 month (Frankel grade E).

\section{DISCUSSION}

Idiopathic SSEHs can be considered rare entities with unsatisfactory functional outcomes in the majority of cases. Postoperative mortality is generally estimated to be $\sim 5 \%{ }^{2}$ Conservative treatment remains an option only in few cases with mild, spontaneously reversing deficits. In most cases, an urgent decompressive laminectomy is indispensable. Precipitating factors related to a poor prognosis include (a) complete neurological deficit; (b) fast progression interval $(<12 \mathrm{~h})$; (c) the involvement of four or more vertebral levels; and (d) edema visible on magnetic resonance imaging. ${ }^{7}$ The operative interval, that is the time between clinical onset and surgical treatment, is considered the most important determining factor that can influence the outcome. Shin et al..$^{8}$ documented in their series of 14 patients a positive functional postoperative recovery, quantified with the Japanese Orthopedic Association score, in $83 \%$ of patients operated within $12 \mathrm{~h}$ from clinical onset, in $63.6 \%$ of those operated between 12 and $24 \mathrm{~h}$ and in $46.7 \%$ of those operated after $24 \mathrm{~h}^{6}{ }^{6}$

Our patient was affected by an extremely rare case of SSEH of idiopathic origin. The hematoma was particularly extensive, involving 10 metameric levels, being, to the best of our knowledge, the second most extensive idiopathic spontaneous hematoma and the longest of the dorso-lumbar tract ever reported. Interestingly, this is also the largest SSEH reported in the vertebral tract that evolved in the absence of any known risk factors and achieved an excellent neurological recovery from Frankel grade B to Frankel grade E. This report emphasizes as a major prognostic factor for patients suffering from SSEH the short operative interval, more than significant 
extension of the hematoma. For this reason early emergency decompressive surgery is recommended in order to ensure satisfactory neurological outcome with almost complete recovery.

\section{CONFLICT OF INTEREST}

The authors declare no conflict of interest.

1 Jackson R. A case of spinal apoplexy. Lancet 1869; 2: 5-6.

2 Kreppel D, Antoniadis G, Seeling W. Spinal hematoma: a literature survey with meta-analysis of 613 patients. Neurosurg Rev 2003; 26: 1-49.
3 Lo C, Chen J, Lo Y, Lai P, Lin Y. Spontaneous spinal epidural hematoma: a case report and review of the literatures. Acta Neurol Taiwan 2012; 21: 31-34.

4 Lonjon MM, Paquis P, Chanalet S, Grellier P. Nontraumatic spinal epidural hematoma: report of four cases and review of literature. Neurosurgery 1997; 41: 483-487.

5 Groen RJ, van Alphen HA. Operative treatment of spontaneous spinal epidural hematomas: a study of the factors determining postoperative outcome. Neurosurgery 1996; 39: 494-5096.

6 Lawton MT, Porter RW, Heiserman JE, Jacobowitz R, Sonntag VK, Dickman CA Surgical management of spinal epidural hematoma: relationship between surgical timing and neurological outcome. J Neurosurg 1995; 83: 1-7.

7 Zhong W, Chen H, You C, Li J, Liu Y, Huang S. Spontaneous spinal epidural hematoma. J Clin Neurosci 2011; 18: 1490-1494.

8 Shin JJ, Kuh SU, Cho YE. Surgical management of spontaneous spinal epidural hematoma. Eur Spine J 2006; 15: 998-1004. 